\title{
Ebbinghaus illusions: Size contrast or contour interaction phenomena?
}

\author{
TED JAEGER \\ University of Georgia, Athens, Georgia 30602
}

\begin{abstract}
Forty-eight graduate and undergraduate students between 17 and 30 years of age judged the size of the central circle in Ebbinghaus figures presented under either simultaneous or successive viewing conditions. Successive presentation eliminated the illusion produced by the normally overestimated figure and diminished that produced by the underestimated figure. Under simultaneous viewing conditions, increasing the number of surrounding circles reduced the magnitude of the reversed illusion produced by the normally overestimated figure but had no effect on the illusion produced by the underestimated figure. Under successive viewing conditions, increasing the number of surrounding circles had no effect on the magnitude of illusion produced by the normally overestimated figure but increased the illusion produced by the underestimated figure. These data were interpreted as inconsistent with a size contrast explanation of the Ebbinghaus illusions and suggested that these illusions may be produced by a contour interaction process.
\end{abstract}

Massaro and Anderson (1971) have presented an explanation of the Ebbinghaus illusion based on a cognitive size contrast mechanism. Briefly, their account of the illusion suggests that in both overestimated and underestimated Ebbinghaus figures, judgments of the size of the central circle are made in relation to the size of the surrounding circles. When the surrounding circles are small, the contrast mechanism causes an increment in the apparent size of the central circle; when the surrounding circles are large, the contrast mechanism causes a decrement in the apparent size of the central circle. The data presented by Massaro and Anderson (1971) in support of their theory show that the magnitude of this size contrast effect varies directly with both the number of surrounding circles and the size difference between the surrounding and central circles. Also, for the overestimated Ebbinghaus figure, they demonstrate that the strength of the contrast effect varies inversely with the separation of surrounding and central circles.

Although these findings are consistent with a size contrast explanation of the Ebbinghaus illusion, more recent studies present difficulties for this theory. Using the underestimated Ebbinghaus figure, Girgus, Coren, and Agdern (1972) found that increasing the separation of surrounding and central circles increased illusion magnitude. Also, they found that, with large separations of surrounding and central circles, the direction of the normally overestimated Ebbinghaus illusion was reversed. Jaeger and Pollack (1977) have shown that increasing the ratio of the lightness contrast of the surrounding to the central circles of both overestimated and underestimated Ebbinghaus figures increases the apparent size of the central circle. The latter finding suggests that a contour interaction process must be at least partially responsible for both components of the Ebbinghaus illusion.

Aside from providing data which are inconsistent with the size contrast theory, the aforementioned studies also point out some similarities between the Delboeuf and Ebbinghaus illusions. For instance, for the Delboeuf illusion, small separations of outer and inner circles produce overestimation, whereas large separations produce underestimation (Ikeda \& Obonai, 1955; Keats, 1964). For the Ebbinghaus illusion, when the contours composing the ring of surrounding circles are close to the central circle, the size of the central circle is overestimated; however, when the contours composing the ring of surrounding circles are made more distant from the central circle, either by increasing the size of the surrounding circles or by increasing the separation of the surrounding and central circles, the size of the central circle is underestimated (Girgus, Coren, \& Agdern, 1972). Also, for the Delboeuf figure, increasing the lightness contrast of the outer circle relative to that of the inner circle increases the apparent size of the inner circle (Oyama, 1962; Weintraub, Wilson, Greene, \& Palmquist, 1969). For the Ebbinghaus figures, increasing the lightness contrast of the surrounding circles relative to the central circle increases the apparent size of the central circle (Jaeger \& Pollack, 1977). Collectively, these studies suggest that the Delboeuf and Ebbinghaus illusions may simply be variants of each other with the ring of surrounding circles distorting the apparent size 
of the central circles of the Ebbinghaus figure in the same manner as the outer circle distorts the apparent size of the inner circle in the Delboeuf figure.

If, in fact, the Delboeuf and Ebbinghaus illusions are variants of each other, it should be possible to show that corresponding alterations in any stimulus variables affect the magnitude of both illusions similarly. Two variables which are known to affect the direction and magnitude of the Delboeuf illusion are presentation sequence and the amount of contour composing the outer and inner circles of the Delboeuf figure. Ikeda and Obanai (1955) have shown that presenting the outer and inner circles of both overestimated and underestimated simultaneous Delboeuf figures successively produces an underestimation of inner circle size. Weintraub, Wilson, Greene, and Palmquist (1969) have found that, for simultaneous Delboeuf figures having portions of either the outer or inner circle removed, the illusion of inner circle overestimation grows in strength as the ratio of the contour present in the outer to the inner circle increases. Thus, assuming that the Delboeuf and Ebbinghaus illusions are variants of each other with the ring of surrounding circles of the Ebbinghaus figure distorting the size of the central circle just as the outer circle of the Delboeuf figure distorts the size of its inner circle, it can be predicted that successive presentation of the surrounding and central circles of the Ebbinghaus figures should produce an underestimation of central circle size. Note that, for the overestimated Ebbinghaus figure, this implies a reversal in the direction of the illusion to underestimation. Also, if changes in the amount of contour composing the outer circle of the Delboeuf figure are analogous to changes in the number of circles in the ring of surrounding circles of the Ebbinghaus figures, it could be expected that increasing the number of surrounding circles would increase the strength of the illusion produced by the overestimated and underestimated simultaneous Ebbinghaus figures. Massaro and Anderson's (1971) data verify this expectation and do not, therefore, provide unequivocal support for their size contrast theory. A prediction regarding the effect of the number of surrounding circles on the successive Ebbinghaus illusion is more difficult, since, as yet, no experiment has been reported which examines the effect of outer and inner circle completeness on the successive Delboeuf illusion. However, it can be argued that if the successive Ebbinghaus illusions result from some form of a contour interaction process, as the successive Delboeuf illusion may (Ganz, 1966a), the addition of more inducing contour produced by increasing the number of surrounding circles should serve to increase the underestimation of central circle size.

While the foregoing predictions are derived from a Delboeuf-like contour interaction explanation of the
Ebbinghaus illusion, other predictions follow from the size contrast theory. Massaro and Anderson (1971) have argued that when the surrounding and central circles of the Ebbinghaus figures are presented successively, the saliency of the surrounding circles during the size contrast process is reduced. Therefore, from this theory, it can be predicted that successive presentation should diminish the magnitude, but not reverse the direction, of either the overestimated or underestimated component of the Ebbinghaus illusion. Furthermore, increases in the number of surrounding circles should simply increase the magnitude of overestimation and underestimation irrespective of presentation sequence, since a larger number of surrounding circles provides a more influential context against which the size of the central circle is judged.

This experiment is designed to evaluate the adequacy of the size contrast and contour interaction explanations of the Ebbinghaus illusion by testing these alternative predictions.

\section{METHOD}

\section{Subjects}

The subjects were 24 male and 24 female white college students ranging in age from 17 to 30 years. Each had a visual acuity of $20 / 20$ or better as measured by a Master Orthorater.

\section{Stimuli}

The stimulus figures were drawn on white $5 \times 7$ in. file cards with black (2.7\% reflectance) India ink. For simultaneous viewing, each stimulus card contained either an overestimated or underestimated Ebbinghaus figure with two, four, or six surrounding circles. The diameter of the central circle of all Ebbinghaus figures was $4.8 \mathrm{~mm}$. The diameter of the small surrounding circles was $3.6 \mathrm{~mm}$, and the diameter of the large surrounding circles was $6.0 \mathrm{~mm}$. For both the overestimated and underestimated illusion figures, the separation between the edge of the central circle and the proximal edges of the surrounding circles was $2 \mathrm{~mm}$. Each Ebbinghaus figure was centered $22 \mathrm{~mm}$ to one side of a centrai fixation point and a comparison circle was drawn an equal distance to the side of the fixation point opposite the illusion figure. The diameter of the comparison circles on the cards containing the overestimated Ebbinghaus figures ranged from 3.30 to $6.30 \mathrm{~mm}$ in .25-mm-interval steps; the diameters of the comparison circles on the cards containing the underestimated Ebbinghaus figures ranged from 3.05 to $6.05 \mathrm{~mm}$ in .25 -mm-interval steps. The maximal height of the stimulus array containing the overestimated figures was $0^{\circ} 40^{\prime} 40^{\prime \prime}$, while the width varied from $2^{\circ} 24^{\prime} 20^{\prime \prime}$ to $2^{\circ} 30^{\prime}$. For the underestimated figures, the maximal height of the stimulus array was $0^{\circ} 53^{\prime} 20^{\prime \prime}$ and the width varied from $2^{\circ} 40^{\prime} 40^{\prime \prime}$ to $2^{\circ} 46^{\prime} 40^{\prime \prime}$.

For successive viewing conditions, 12 stimulus cards were constructed containing only the surrounding circles of the Ebbinghaus figures. Six of the cards contained small surrounding circles, $3.60 \mathrm{~mm}$ in diameter, with two, four, or six of these circles centered $22 \mathrm{~mm}$ to either the right or left of a central fixation point. The other six cards contained large surrounding circles, $6.00 \mathrm{~mm}$ in diameter, with two, four, or six of these circles centered $22 \mathrm{~mm}$ to either the right or left of a central fixation point. The central and comparison circles were drawn on a separate set of 12 cards. The central circles were centered $22 \mathrm{~mm}$ to either the right or left of a central fixation point, and the comparison circles were centered an equal distance to the opposite side of the fixation point. The diameters of the comparison circles ranged from 3.05 to $6.05 \mathrm{~mm}$ in .25 -mm-interval steps. 
By pairing the stimulus cards containing only the surrounding circles with the stimulus cards containing the central and comparison circles, successive Ebbinghaus figures with two, four, or six surrounding circles were produced. The visual angle sizes of these successive Ebbinghaus figures matched those of the simultaneous figures.

In addition to the illusion stimuli, control stimuli were constructed consisting of a single test circle, $4.80 \mathrm{~mm}$ in diameter, centered $22 \mathrm{~mm}$ to either the right or left of a central fixation point and a comparison circle centered an equal distance to the opposite side of the fixation point. The diameter of the comparison circles ranged from 3.80 to $5.80 \mathrm{~mm}$ in .25 -mm-interval steps. The maximal height of the stimulus array was $0^{\circ} 15^{\prime} 40^{\prime \prime}$, and the width varied from $2^{\circ} 16^{\prime} 40^{\prime \prime}$ to $2^{\circ} 20^{\prime}$.

\section{Procedure}

The subjects were assigned randomly to one of four experimental groups with the stipulation that an equal number of males and females be represented in each group. Group 1 viewed the overestimated Ebbinghaus figure under conditions of simultaneous presentation; Group 2 viewed the overestimated Ebbinghaus figure under conditions of successive presentation; Group 3 viewed the underestimated Ebbinghaus figure under conditions of simultaneous presentation; Group 4 viewed the underestimated Ebbinghaus figure under conditions of successive presentation.

Before the experimental session, the subjects were dark-adapted for $5 \mathrm{~min}$, during which instructions were given. Each subject was told to respond "larger" when the comparison circle appeared larger than the central circle of the Ebbinghaus figure and "smaller" when the comparison circle appeared smaller than the central circle of the Ebbinghaus figure. No "equal to" responses were accepted.

Stimuli were presented in a three-channel tachistoscope (Scientific Prototype, Model GB). The level of illumination at the eyepiece was $16.5 \mathrm{~mL}$. Under simultaneous viewing conditions, the exposure duration of each illusion figure was $500 \mathrm{msec}$. Under successive conditions, the inducing and test components of the illusion figure were each presented for $500 \mathrm{msec}$ and a $500 \mathrm{msec}$ interstimulus interval intervened between their presentation. Each experimental session began and ended with a control condition in which the subjects estimated the size of a test circle in the Ebbinghaus figures. The control condition contained four trials, each trial consisting of eight judgments. The order of stimulus presentation was determined by a converging method of limits (Clem \& Pollack, 1975), and initiation of each trial with either a large or small comparison circle was counterbalanced with an ABBA design. Also, an ABBA design was used to counterbalance the placement of the test and comparison circles to either the right or left of the central fixation point. After the control trials, the subjects continued, in their respective experimental groups, to judge the size of the central circle in one Ebbinghaus figure under either simultaneous or successive conditions. Repeated measures were taken across the surrounding circles variable, with each subject receiving eight trials per level and 12 judgments per trial. Again, order of stimulus presentation was determined by a converging method of limits, and initiation of trials with either a large or small comparison circle was counterbalanced with an ABBA design. The placement of the Ebbinghaus figure and comparison circle to either the right or left of the fixation point was also counterbalanced following an ABBA design, and a balanced square design was used to determine the order of presentation of the number of surrounding circles conditions for each subject.

\section{RESULTS}

Points of subjective equality (PSEs), defined as the point at which a changed response was maintained for three consecutive judgments, were calculated for each subject on each trial. These scores were converted to illusion magnitude scores by subtracting the mean control PSE from the mean PSE of each experimental condition for each subject. The mean illusion magnitude scores for each of the 12 experimental conditions are presented in Figure 1. Observation of this figure reveals a surprising result. For all Ebbinghaus figures, regardless of whether the surrounding curcles are smaller or larger than the central circle, the size of the central circle was underestimated. Since, in the case of the Ebbinghaus figures with small surrounding circles, this underestimation was of considerable theoretical importance, criterion $t$ tests were conducted to determine if the illusion produced by these figures differed significantly from zero. For the simultaneous figures with either two or four surrounding circles, the underestimation of central circle size was significant. For the simultaneous figure with six surrounding circles, as well as for all three of the successive figures with small surrounding circles, there was no significant amount of inner circle underestimation.

The data presented in Figure 1 were analyzed with a three-factor mixed model analysis of variance, and the results indicated that the main effects of illusion $(\mathrm{F}=8.518, \mathrm{df}=1,44, \mathrm{p}<.01)$ and presentation

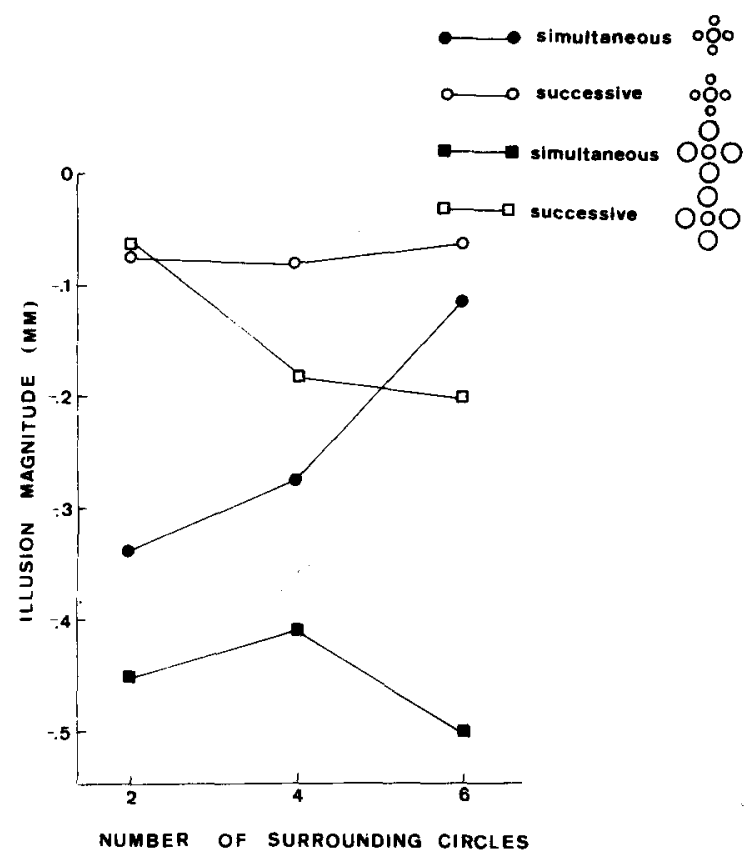

Figure 1. Illusion magnitude as a function of surrounding circle number. Each data point is averaged across 12 subjects with eight converging method-of-limits trials per subject. The negative signs in the graph represent underestimation of central circle size which, in the case of the Ebbinghaus figures with small surrounding circles, is a reversal of the expected central circle overestimation. 
$(\mathrm{F}=21.405$, df $=1,44, \mathrm{p}<.001)$ were both significant. The main effect of surrounding circle number was not significant $(F=.166, \mathrm{df}=2,90$, $\mathrm{p}<.20$ ), although inspection of Figure 1 shows that this effect was averaged out due to the opposing effects of surrounding circle number of the magnitude of illusion produced by the simultaneous normally overestimated figure and the successive underestimated figure.

In addition to these main effects, the Surrounding Circle by Illusion $(\mathrm{F}=11.092, \mathrm{df}=2,90, \mathrm{p}<.001$ ) and Surrounding Circle by Presentation $(F=2.889$, $\mathrm{df}=2,90, \mathrm{p}<.01$ ) interactions were also significant. The Surrounding Circle by Illusion interaction is attributable to the fact that increasing the number of surrounding circles generally seems to decrease the illusion produced by the normally overestimated Ebbinghaus figures while increasing the illusion produced by the underestimated Ebbinghaus figures. The Surrounding Circle by Presentation interaction reflects the opposing effects of variation in the number of surrounding circles on the magnitude of the simultaneous and successive illusions. Collapsing the data across illusion conditions, it can be seen that increasing the number of surrounding circles produces a decrease in the magnitude of the simultaneous illusion while an increase in the magnitude of the successive illusions is observed.

Finally, since there was interest in determining the effects of surrounding circle number within illusion and presentation conditions, the simple effect of surrounding circles at each presentation level of each illusion were evaluated. For the normally overestimated simultaneous illusion, the simple effect of surrounding circles was significant $(\mathrm{F}=11.001$, $\mathrm{df}=2,90, \mathrm{p}<.001$ ). A Newman-Keuls test showed that the magnitude of illusion produced by the figure with six surrounding circles differed significantly from that produced by the figures with either two or four surrounding circles. The effect of surrounding circle number was not significant for either the successive normally overestimated illusion $(\mathrm{F}=$ .060 , $\mathrm{df}=2,90, \mathrm{p}>.20$ ) or for the simultaneous underestimated illusion $(F=1.719$, $\mathrm{df}=2,90$, $p>.20)$. However, for the successive underestimated illusion, the effect of surrounding circle number was significant $(F=4.779$, df $=2,90, p<.05)$. For the latter illusion, a Newman-Keuls test revealed that the magnitude of illusion produced by the figure with two surrounding circles differed significantly from that produced by the illusion figures with either four or six surrounding circles.

\section{DISCUSSION}

The data reported here present further difficulties for a size contrast explanation of the Ebbinghaus illusion, particularly in the case of the normally overestimated figure. Successive presentation of the surrounding and central circles of this figure result in some, albeit insignificant, amount of central circle underestimation. The failure of this illusion to persist under successive viewing conditions argues against the size contrast theory, but more decisive is the fact that the illusion is also reversed under simultaneous viewing conditions. Since the surrounding circles are smaller than the central circle, any size contrast effect would result in overestimation of the size of the central circle, not the underestimation found here. It is also important to note that the amount of central circle underestimation decreases as the number of surrounding circles is increased. Weintraub, Wilson, Greene, and Palmquist (1969) have reported an analogous finding for the Delboeuf illusion. That is, if portions of the contour of the outer circle of the Delboeuf figure are deleted so that only $25 \%$ of the inducing contour remains, the overestimation of central circle size turns to underestimation. With addition of inducing contour so that the outer circle is $50 \%$ present, the illusion reverts to overestimation, and overestimation grows stronger as the remaining portions of the inducing contour are added. Thus, if the surrounding circles of the normally overestimated Ebbinghaus figure function analogously to the outer circle of the Delboeuf figure, it would seem that further addition of surrounding circles to the normally overestimated Ebbinghaus figure would ultimately produce overestimation of the size of the central circle.

With respect to the underestimated Ebbinghaus illusion, the data are somewhat more difficult to interpret. Underestimation of the central circle persists when the surrounding and central circles are presented successively. This finding is consistent with both the size contrast and contour interaction interpretations of the illusion. The same equivocality is present in the data showing that increasing the number of surrounding circles in this successive figure increases the amount of central circle underestimation. This result could be due either to a more influential ground against which the size of the central circle is judged or the increasing amount of inducing contour present which acts to diminish the apparent size of the central circle through an afterimage process. The latter interpretation receives some support from O'Hallaron and Weintraub's (1977) recent demonstration that afterimages of the surrounding circles of this illusion figure do produce underestimation of central circle size when viewed with the central circle simultaneously present. The absence of a size contrast effect in the underestimated illusion is more convincingly demonstrated by the failure of increases in the number of surrounding circles to increase the illusion strength when the 
underestimated figure is presented under simultaneous viewing conditions. This result contradicts not only Massaro and Anderson's (1971) theory, but also their data which ostensibly show an increasing size contrast effect with a larger number of surrounding circles. The reasons for not finding this effect in the underestimated simultaneous illusion in this experiment are not clear, though a possible explanation will be discused below.

In general, the results presented here cast doubt on the validity of a size contrast explanation of the Ebbinghaus illusion. Furthermore, they point out additional similarities between the misestimations of circle size occurring in the Ebbinghaus and Delboeuf figures. Since the Delboeuf illusion has typically been regarded as the outcome of an interaction between the contours of the outer and inner circles, the accumulation of data indicating that this and the Ebbinghaus illusion are merely variants of each other suggests that the latter illusion also results from a contour interaction process. However, since the data presented here show differential effects of surrounding circle number for the simultaneous and successive versions of both the normally overestimated and underestimated Ebbinghaus illusions, it is apparent that different contour interaction processes must be activated by the two means of presentation. For both successive Ebbinghaus illusions, the fact that there is some degree of underestimation of the inner circle indicates that the contour of the inner circle must have been shifted away from the locus of the contour of the surrounding circles. Such a shift could have been produced by a satiation of the cortical region where the surrounding circles were presented (Kohler \& Wallach, 1944) or by lateral inhibitory interactions between the neural trace of the afterimage of the surrounding circles and the physically present inner circle (Ganz, 1966b). However, the latter alternative seems more plausible, given O'Hallaron and Weintraub's (1977) demonstration that Ebbinghaus figures formed by the visible afterimage of large outer circles and a physically present inner circle produce inner circle underestimation. This finding implies that the trace of an afterimage could be expected to produce contour displacements in a direction which account for the underestimation of the size of the inner circles in the successive Ebbinghaus figures employed here.

The data are also of value in choosing between different contour interaction theories which might account for the misestimations of central circle size occurring in the simultaneous Ebbinghaus illusions. There are, at present, three formulations of contour interaction theory which could be applicable. Pollack (1964) has claimed that simultaneous contour interaction effects depend on the mutual orientation of contours; parallel contours attract and orthogonally oriented contours repel. Ganz (1966b) has claimed that all spatially adjacent contours, regardless of orientation, should repel each other due to lateral inhibitory interactions between adjacent retinal cells coding the contour's spatial location. On the other hand, Brigner (1977) attributes simultaneous contour interaction effects to facilitory and inhibitory interactions between cortical units coding contour length. Facilitory interactions are said to occur when inducing and test contours are only slightly separated; inhibitory interactions predominate at larger separations. Both Pollack's (1964) and Ganz's (1966b) theories can predict only underestimation of central circle size in the Ebbinghaus illusion, since the contours composing the surrounding circles are closely adjacent and, for the most part, oriented orthogonally to those of the central circle, While it is true that only underestimation of the central circle size was found in this experiment, Jaeger and Pollack (1977) found, under identical viewing conditions, overestimation of central circle size for the simultaneous Ebbinghaus figure with small surrounding circles. This shift from central circle overestimation to underestimation seems to have resulted from a modification in the dimensions of the Ebbinghaus figure. Specifically, in this study, the normally overestimated Ebbinghaus figure had both larger surrounding circles and a smaller central circle. Since one of the consequences of increasing the size of the small surrounding circles is to make a larger portion of their contour more distant from the central circle, Brigner's (1977) theory offers a possible explanation for the appearance of central circle underestimation in the normally overestimated Ebbinghaus figure used here. Additional support for the notion that contour separation is the critical variable determining the direction of the Ebbinghaus illusion comes from Japanese studies reviewed by Oyama (1960). These studies show that deleting the outer portions of the larger circles of simultaneous underestimated Ebbinghaus figures changes the illusion to overestimation while deleting the inner portions of the same surrounding circles fail to reduce the amount of central circle underestimation.

From these data it appears that the inner and outer portions of the surrounding circles of the underestimated Ebbinghaus figure have antagonistic effects on the apparent size of the center circle; the outer portions of the surrounding circles seem to generate underestimation, while the inner portions seem to produce overestimation. These facts are consistent with Brigner's (1977) account of simultaneous contour interaction effects and suggest an explanation for this study's finding that increases in the number of surrounding circles do not significantly alter the magnitude of the simultaneous under- 
estimated Ebbinghaus illusion. Specifically, it seems the size of the illusion figures is such that the addition of surrounding circles adds contours responsible for both overestimation and underestimation in equal proportions. Since Brigner's (1977) theory provides the most parsimonious account of these contour interaction effects, it appears to provide the most satisfactory explanation of the simultaneous Ebbinghaus illusion presently available.

\section{REFERENCES}

Brugner, W. L. Mathematical model for assimilation and contrast in the perception of extent. Perceptual and Motor Skills, 1977, 45, 103-118.

Clem, R. K., \& Pollack, R. H. Illusion magnitude as a function of visual field exposure. Perception \& Psychophysics, 1975, 17, 450-454.

GANZ, L. Is the figural aftereffect an after effect? A review of its intensity; onset, decay, and transfer characteristics. Psychological Bulletin, 1966, 66, 151-165. (a)

Ganz, L. Mechanism of the figural aftereffect. Psychological Review, 1966, 73, 128-150. (b)

Girgus, J. S., Coren, S., \& Agdern, M. The interrelationship between the Ebbinghaus and Delboeuf illusions. Journal of Experimental Psychology, 1972, 95, 453-455.

IKeDA, H., \& ObanaI, T. Studies in figural after-effects (IV): The contrast-confluence illusion of concentric circles and the figural after-effect. Japanese Psychological Research, 1955, 1, 17-23.
Jaeger, T, \& Pollack, R. H. Effect of contrast level and temporal order on Ebbinghaus circles illusion. Perception \& Psychophysics, 1977, 21, 83.87.

KeATs, J. A. An application of the study of paired comparisons to the study of the Delboeuf illusion. Australian Journal of Psychology, 1964, 16, 169-174.

Kohler, W., \& Wallach, H. Figural after-effects: An investigation of visual processes. Proceedings of the American Philosophicai Society, 1944, 88, 316-357.

Massaro, D. W., \& Anderson, N. H. Judgmental model of the Ebbinghaus illusion. Journal of Experimental Psychology, 1971, 89, 147-151.

O'Hallaron, W. J., \& Weintraub, D. J. Delboeuf "illusions" of circle size induced via photoflash-generated afterimages. Perception \& Psychophysics, 1977, 22, 171-176.

Oyama, T. Japanese studies on the so-called geometrical optical illusions. Psychologia, 1960, 3, 7-20.

Oyama, T. The effect of hue and brightness on the sizeillusion of concentric circles. American Joumal of Psychology, 1962, 75, 45-55.

Pollack, R. H. The effect of fixation upon the apparent magnitude of bounded horizontal extent. American Journal of Psychology, 1964, 77, 177-192.

Weintraub, D. J., Wilson, B. A., Greene, R. D., \& PalmQuss, M. J. Delboeuf illusion: Displacement versus diameter, arc deletions, and brightness contrast. Journal of Experimental Psychology, 1969, 80, 505-511.

(Received for publication March 20, 1978; revision accepted July 18,1978 .) 